\title{
Effect of Induced Magnetic Field on Natural Convection in Vertical Concentric Annuli Heated/Cooled Asymmetrically
}

\author{
A. $\operatorname{Kumar}^{1 \dagger}$ and A. K. Singh ${ }^{2}$ \\ ${ }^{I}$ Department of Mathematics, Central University of Rajasthan, Ajmer, 305801, India \\ ${ }^{2}$ Department of Mathematics, Faculty of Science, Banaras Hindu University, Varanasi, 221005, India
}

†Corresponding Author Email: aanandbhu@gmail.com

(Received June 28, 2010; accepted July 12, 2011)

\begin{abstract}
In the present paper, the fully developed laminar free convective flow of a viscous incompressible and electrically conducting fluid between two concentric vertical cylinders is considered in the presence of a radial magnetic field. The induced magnetic field produced by the motion of an electrically conducting fluid is taken into account. The expressions for the temperature, velocity, induced magnetic field, induced current density, skin-friction and Nusselt number are obtained in a closed form under more general boundary conditions for the induced magnetic field. The influence of the Hartmann number and buoyancy force distribution parameter on the fluid velocity, induced magnetic field and induced current density have been analyzed by using the graphs while the values of the skin-friction, Nusselt number, induced current flux and mass flux are given in the tabular form. It is observed that the fluid velocity and induced magnetic field are rapidly decreasing with increase in the value of Hartmann number in the case when one of the cylinders is conducting compared with the case when both cylinders are non-conducting. The effect of the induced magnetic field is to increase the velocity profiles in comparison to the case of neglecting the induced magnetic field. The buoyancy force distribution parameter has tendency to increase the fluid velocity, induced magnetic field, temperature field and induced current flux.
\end{abstract}

Keywords: Induced magnetic field, Free convection, Current flux, Current density, Hartmann number, Buoyancy force distribution

\section{NOMENCLATURE}

$\begin{array}{ll}a & \begin{array}{l}\text { radius of inner cylinder } \\ \text { radius of outer cylinder }\end{array} \\ g & \begin{array}{l}\text { acceleration due to gravity } \\ \text { constant magnetic field }\end{array} \\ H_{0} & \begin{array}{l}\text { induced magnetic field component along } \\ \text { the axis of cylinder }\end{array} \\ h^{\prime} & \begin{array}{l}\text { dimensionless induced magnetic field } \\ \text { component along the axis of the cylinder }\end{array} \\ h & \text { characteristic velocity } \\ U & \text { Hartmann number } \\ M & \text { Nusetl number at inner cylinder } \\ N u_{1} & \text { Nusetl number at outer cylinder } \\ N u_{\lambda} & \text { buoyancy force distribution } \\ R & \text { radial distance } \\ r & \text { dimensionless radial distance } \\ r & \text { dimensionless temperature } \\ T & \text { temperature of the inner cylinder }\end{array}$
$T_{b}^{\prime} \quad$ temperature of the outer cylinder
$T_{f}^{\prime} \quad$ temperature of the fluid
$u^{\prime} \quad$ velocity component along the axis of
cylinder
$u \quad$ dimensionless velocity component along the axis of cylinder

\section{Greek symbols}

$\begin{array}{ll}\beta & \text { coefficient of thermal expansion } \\ \mu & \text { coefficient of viscosity } \\ \mu_{e} & \text { magnetic permeability } \\ v & \text { kinematic viscosity of the fluid } \\ \rho & \text { density of the fluid } \\ \sigma & \text { electrical conductivity } \\ \tau_{1} & \text { skin friction coefficient at inner cylinder } \\ \tau_{\lambda} & \text { skin friction coefficient at outer cylinder }\end{array}$




\section{INTRODUCTION}

The studies of free-convective flow along a vertical cylinder are important due to its applications in the field of geothermal power generation, drilling operations, geological formulation. Globe (1959) investigated the steady flow of an electrically conducting fluid in the annular space between two infinite long circular cylinders under the presence of a radial magnetic field. Dube (1971) considered the steady laminar flow of a viscous incompressible and electrically conducting fluid between infinitely long concentric rotating porous cylinders under the influence of a radial magnetic field. Pop et al.(1993) investigated the growth of the free convection boundary-layer on a isothermal horizontal circular cylinder embedded in a porous medium while Tyvand [4] have extend the work of Pop et al. (1993) by considering the transient convection in a porous medium, due to sudden temperature change at a boundary. Seong and Choung (2001) have investigated the flow past a circular cylinder under continuous and pulsed electromagnetic forces.

El-Shaarawi and Sarhan (1981) have considered the fully developed free convective flow in the vertical annuli with one boundary isothermal and opposite adiabatic boundary while Joshi (1987) has taken the isothermal boundaries in which the inner boundary is maintained at a higher temperature than the outer one. Lien et al. (1985) have shown the effect of heat transfer on free convective flow as a result of impulsive motion of an infinite vertical circular cylinder. They have considered the isothermal and constant heat flux cases for oscillating and impulsively started circular cylinder while El-Shaarawi et al. (1990) have studied the fully developed laminar natural convection by considering four fundamental boundary conditions. These boundary conditions are obtained by combining each of the two conditions having one boundary maintained at uniform heat flux or at uniform wall temperature with each of the conditions while the opposite boundary conditions is kept isothermal at the cove fluid temperature or adiabatic. Several authors (see Sastry et al.(1987); Singh et al.(1997); Paul et al.(1998); Yan et al.(2000)) have investigated the convective heat transfer in hydromagnetics in view of its utility in exploration, thermal recovery of oil and underground nuclear waste storage site. Sastry and Bhadram (1987) have considered the combined free and forced convective flow and heat transfer in vertical annulus when a radial magnetic field is applied. Singh et al. (1997) studied the fully developed natural convective flow in the presence of a radial magnetic field by obtaining a unified solution when the thermal boundary condition at the inner cylinder is of mixed kind while outer one is kept on constant temperature. Paul and Singh (1998) have shown the analytical solution of laminar fully developed free convective flow between two coaxial vertical cylinders partially filled with a porous medium and clear fluid. Yan (2000) has considered viscous flow about a submerged circular cylinder, with oscillates otherwise steady current. Several solutions in the case of hydromagnetic freeconvective flows have been obtained by Chandran et al. (1993, 1996, 1998, 2001) for different physical situations of flow formations.
The above studies on natural convective heat and mass transfer phenomena in different cylindrical geometries in the presence of a magnetic field have been limited to the case when the induced magnetic field is not taken into account. This is due to the fact that the mathematical description as well as solution of such problems involves some less effort. Thus, the main aim of this paper is to present the fully developed free-convective flow of a viscous incompressible fluid between two coaxial vertical cylinders by considering into account the induced magnetic field. Boundary conditions at inner and outer cylinders are of mixed kind and convection between the vertical cylinders is set up by a change in the temperature of the cylinders as compared to fluid temperature. Here we have considered the three cases on boundary conditions of the induced magnetic field in which first case is when both cylinders are non-conducing. In second case, the inner cylinder is taken as non-conduction while outer cylinder as conducting and finally in third case, the inner cylinder is taken as conducting while outer cylinder as non-conducting. A non-dimensional parameter is used in order to characterize the temperature of the concentric cylinder with respect to the fluid temperature. This parameter also characterizes distribution of the buoyancy force between the cylinders and thus provides a very convenient and generalized framework to study the effect of changing the cylinder temperature. An integrated solution has been obtained using the mixed boundary conditions for the induced magnetic field. Finally the effects of buoyancy force distribution and Hartmann number parameter have been shown in the graphical and tabular from.

\section{Mathematical Formulation}

The fully developed natural convective flow of a viscous incompressible and electrically conducting fluid inside vertical concentric annuli of infinite length is considered in which temperature of the fluid as well as temperature of the cylinders are taken different from each other. The $z^{\prime}$-axis is considered along the axis of cylinders in the vertical upward direction and $r^{\prime}$ is in the radial direction measured outward from the axis of the cylinders. The radii of inner and outer cylinders are taken as $a$ and $b$ respectively. The temperature of the fluid is taken $T_{f}^{\prime}$ while the temperatures of inner and outer cylinders are $T_{a}^{\prime}$ and $T_{b}^{\prime}$ respectively. Also the applied induced magnetic field is directed radially outward in the form of $a H_{0} / r^{\prime}$. Since the flow is fully developed and cylinders are of infinite length, the transport phenomena will depend only on $r^{\prime}$. Thus under the usual Boussinesq approximation, the basic transport equations for the considered model are obtained as follows:

$$
\begin{aligned}
& v\left(\frac{\partial^{2} u^{\prime}}{\partial r^{\prime 2}}+\frac{1}{r^{\prime}} \frac{\delta u^{\prime}}{\delta r^{\prime}}\right)+\frac{\mu_{e}}{\rho} \frac{H_{0} a}{r^{\prime}} \frac{\delta h^{\prime}}{\delta r^{\prime}}+g \beta\left(T^{\prime}-T_{m}^{\prime}\right)=0 \\
& \left(\frac{\partial^{2} h^{\prime}}{\partial r^{\prime 2}}+\frac{1}{r^{\prime}} \frac{\delta h^{\prime}}{\delta r^{\prime}}\right)+\frac{\sigma \mu_{e} H_{0} a}{r^{\prime}} \frac{\delta u^{\prime}}{\delta r^{\prime}}=0
\end{aligned}
$$


$\frac{\partial^{2} T^{\prime}}{\partial r^{\prime 2}}+\frac{1}{r^{\prime}} \frac{\delta T^{\prime}}{\delta r^{\prime}}=0$

According to considered model, the boundary conditions for the velocity, induced magnetic field and temperature field are obtained as follows:

$u^{\prime}=0, \frac{\delta h^{\prime}}{\delta r^{\prime}}=0$ or $h^{\prime}=0, T^{\prime}=T_{a}^{\prime}$, at $r^{\prime}=a$

$u^{\prime}=0, \frac{\delta h^{\prime}}{\delta r^{\prime}}=0$ or $h^{\prime}=0, T^{\prime}=T_{b}^{\prime}$, at $r^{\prime}=b$

By using the following non-dimensional variables

$u=\frac{u^{\prime}}{U}, \quad r=\frac{r^{\prime}}{a}, \quad \lambda=\frac{b}{a}, \quad h=\frac{h^{\prime}}{\sigma \mu_{e} H_{0} U a}$

$U=\frac{g \beta a^{2}\left(T_{a}^{\prime}-T_{f}^{\prime}\right)}{v}, T=\frac{T^{\prime}-T_{f}^{\prime}}{T_{a}^{\prime}-T_{f}^{\prime}}$

Eqs. (1) to (3) in dimensionless form are obtained as follows:

$$
\begin{aligned}
& \frac{\partial^{2} u}{\partial r^{2}}+\frac{1}{r} \frac{\delta u}{\delta r}+\frac{M^{2}}{r} \frac{\delta h}{\delta r}+T=0 \\
& \frac{\partial^{2} h}{\partial r^{2}}+\frac{1}{r} \frac{\delta h}{\delta r}+\frac{1}{r} \frac{\delta u}{\delta r}=0 \\
& \frac{\partial^{2} T}{\partial r^{2}}+\frac{1}{r} \frac{\delta T}{\delta r}=0
\end{aligned}
$$

The boundary conditions in the non-dimensional form become

$u=0, \frac{\delta h}{\delta r}=0$ or $h=0, T=1$, at $r=1$

$u=0, \frac{\delta h}{\delta r}=0$ or $h=0, T=R$, at $r=\lambda$

In the above equations $R$ and $M$ are the buoyancy force distribution parameter and the Hartmann number respectively and they are defined by

$R=\frac{T_{b}{ }^{\prime}-T_{f}^{\prime}}{T_{a}{ }^{\prime}-T_{f}^{\prime}}, \quad M=\mu_{e} H_{0} a \sqrt{\frac{\sigma}{\mu}}$

The buoyancy force distribution parameter $R$ will play an important roll on the transport processes between the cylinders. This parameter in actual fact fixes the direction of the fluid temperature $T_{f}^{\prime}$ with respect to cylinders temperature $T_{a}^{\prime}$ and $T_{b}^{\prime}$. When $R=0$ and 1 , then the cases $T_{f}^{\prime}=T_{b}^{\prime}$ and $T_{b}^{\prime}=T_{a}^{\prime}$ are obtained respectively.

When $R$ is in the range $0<R<1$, the case is either $T_{f}^{\prime}<T_{b}^{\prime}<T_{a}^{\prime}$ or $T_{b}^{\prime}<T_{a}^{\prime}<T_{f}^{\prime}$. The case $T_{b}^{\prime}<T_{f}^{\prime}<T_{a}^{\prime}$ results for $R<0$.

A unified solution corresponding to the three cases of boundary conditions on the induced magnetic field can be obtained by combining all conditions for the induced magnetic field. By doing so, the combined conditions are obtained as follows:

$A_{1} \frac{\delta h}{\delta r}+B_{1} h=0$ at $r=1$

$A_{2} \frac{\delta h}{\delta r}+B_{2} h=0$ at $r=\lambda$

The above equation clearly shows that a desired case can be obtained by assigning suitable values to $A_{1}, B_{1}, A_{2}$ and $B_{2}$.

The analytical solutions of Eqs. (6) to (8) subject to their appropriate boundary conditions are obtained as follows:

$$
\begin{aligned}
u= & A+B r^{M}+C r^{-M}+D_{2} r^{2} \log r+D_{3} r^{2} \\
h= & P+S \log r-A \log r-\frac{B}{M} r^{M}+\frac{C}{M} r^{-M}+ \\
& \left(\frac{D_{2}-2 D_{2}}{4}\right) r^{2}-\frac{D_{2}}{2} r^{2} \log r
\end{aligned}
$$

$T=1+D_{1} \log r$

By using the Eqs. (12) and (14) the skin-frictions and the Nusselt number at outer surface of inner cylinder and inner surface of outer cylinder are derived as

$$
\begin{array}{r}
\tau_{1}=\left.\frac{\delta u}{\delta r}\right|_{r=1}=M B-M C+\left(D_{2}+2 D_{3}\right) \\
\tau_{\lambda}=\left.\frac{\delta u}{\delta r}\right|_{r=\lambda}=M B \lambda^{M-1}-M C \lambda^{-M-1}+ \\
\left(D_{2}+2 D_{3}\right) \lambda+2 D_{2} \lambda \log \lambda
\end{array}
$$

$N u_{1}=-\left.\frac{\delta T}{\delta r}\right|_{r=1}=\frac{1-R}{\log \lambda^{\prime}}$

$N u_{\lambda}=-\left.\frac{\delta T}{\delta r}\right|_{r=\lambda}=\frac{1-R}{\lambda \log \lambda^{\prime}}$

The mass flux of fluid, induced current density and induced current flux, by using the Eqs. (12) and (13), are obtained as follows:

$$
\begin{aligned}
& Q=2 \pi \int_{1}^{\lambda} u r d r=2 \pi\left[\frac{A}{2}\left(\lambda^{2}-1\right)+\frac{B}{2+M}\left(\lambda^{2+M}-1\right)\right. \\
& \left.+\frac{C}{2-M}\left(\lambda^{2-M}-1\right)+\left(\frac{4 D_{3}-D_{2}}{16}\right)\left(\lambda^{4}-1\right)+\frac{D_{2}}{4} \lambda^{4} \log r\right] \\
& J_{\theta}=-\frac{d h}{d r}=\frac{A-S}{r}+B r^{M-1}+C^{-M-1}+D_{2} r \log r+D_{3} r \\
& J=\int_{1}^{\lambda} J_{\theta} d r=(A-S) \log r+\frac{B}{M}\left(\lambda^{M}-1\right) \\
& -\frac{C}{M \lambda^{M}}\left(1-\lambda^{M}\right)-\frac{\left(D_{2}-2 D_{3}\right)}{4}\left(\lambda^{2}-1\right)+\frac{D^{2}}{2} \lambda^{2} \log \lambda
\end{aligned}
$$

where $A, B, C, P, S, D_{1}, D_{2}, D_{3}$ are constants and they are defined in Appendix A. 


\subsection{Solution for $\mathrm{M}=2$}

It can be observed from Eqs. (12) and (13) that the solutions for velocity and induced magnetic field are singular at $M=2.0$ and, therefore, we have derived separately the expressions for the velocity and induced magnetic field from Eqs. (7) and (8) by taking $M=2.0$ and they are as follows:

$$
\begin{aligned}
u= & E+F r^{2}+G r^{-2}+D_{26} r^{2}+D_{27} r^{2} \log r+ \\
& D_{28} r^{2}(\log r)^{2} \\
h= & K+L \log r-E \log r-\frac{F}{2} r^{2}+\frac{G}{2} r^{-2}- \\
& D_{46} r^{2}-D_{47} r^{2} \log r-D_{48} r^{2}(\log r)^{2}
\end{aligned}
$$

By using the Eq.(22), the skin-friction at cylindrical walls is obtained as

$$
\begin{aligned}
& \tau_{1}=2 F-2 G+\left(2 D_{26}+D_{27}\right) \\
& \tau_{\lambda}=2 F \lambda-2 G \lambda^{-3}+\left(2 D_{26}+D_{27}\right) \lambda+ \\
& \quad\left(2 D_{27}+2 D_{28}\right) \lambda \log \lambda+2 D_{28} \lambda(\log \lambda)^{2}
\end{aligned}
$$

and the mass flux, induced current density and induced current flux are given by

$$
\begin{aligned}
& Q=\frac{\pi}{16}\left[32 G \log \lambda+16 E\left(\lambda^{2}-1\right)+\right. \\
& \left(8 F+8 D_{26}-2 D_{27}+D_{28}\right)\left(\lambda^{4}-1\right)+ \\
& \left.\left(8 D_{27}-4 D_{28}+8 D \log \lambda\right) \lambda^{4} \log \lambda\right] \\
& J_{\theta}=\frac{E-L}{r}+F r+G r^{-3}+\left(2 D_{46}+D_{47}\right) r+ \\
& 2\left(D_{47}+D_{48}\right) r \log r+2 D_{48} r(\log \lambda)^{2} \\
& D_{52}=D_{50}+D_{51}, D_{53}=\frac{A_{2}}{\lambda}+B_{2} \log \lambda, \\
& D_{54}=B_{2} D_{49}-B_{1} D_{52}, D_{55}=A_{1} B_{2}-B_{1} D_{55} \\
& D_{56}=D_{49} D_{53}-A_{1} D_{52}, K=-\frac{D_{56}}{D_{55}}, L=\frac{D_{54}}{D_{55}}
\end{aligned}
$$

The constants $E, F, G, K, L, D_{26}, D_{27}, D_{28}, D_{46}, D_{47}$, $D_{48}$ appearing in the above equations are defined in Appendix B.

\section{RESUlTS AND DisCUSSION}

The analytical solution obtained in the present investigation is used to carry out a number of simulations for different values of the controlling parameters such as the buoyancy force distribution parameter and Hartmann number while keeping the ratio of radii as 2.0. The simulated numerical values are illustrated by using the figures and tables in order to examine the behavior of buoyancy force distribution parameter and Hartmann number on the transport processes. The temperature profiles are shown in Figs. 1 and 2 for the cases $0 \leq R \leq 1$ and $R<0$ respectively. These figures reveal that the temperature profiles have increasing tendency with $\mathrm{R}$ and then finally by increasing takes the shape of a straight line.

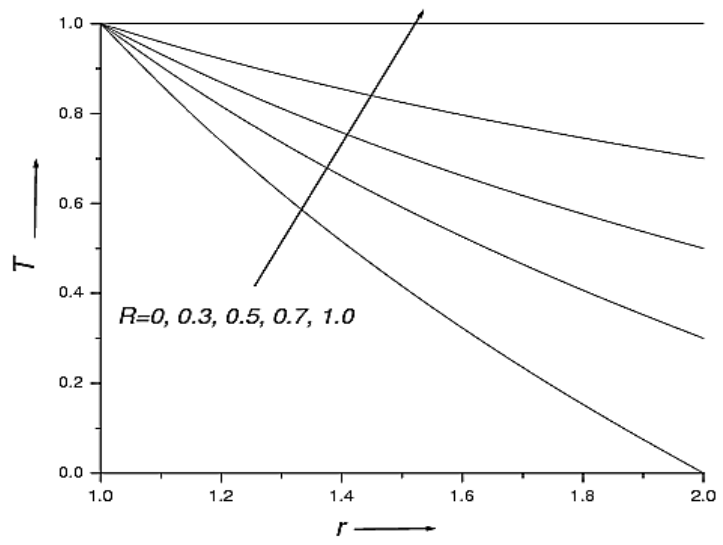

Fig. 1. Temperature profile when $0 \leq R \leq 1$ and

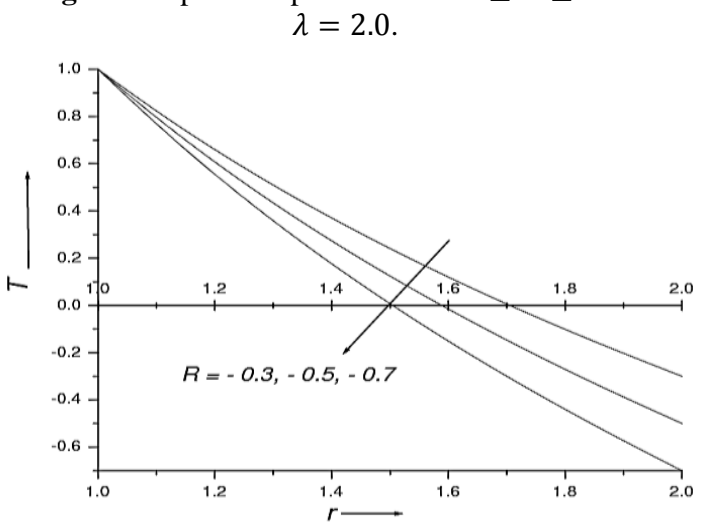

Fig. 2: Temperature profile when $R<0$ and $\lambda=2.0$.

The velocity profiles are plotted in Fig. 3 for the case $0 \leq R \leq 1$ when both cylinders are non-conducting. We scrutinized that the maximum velocity for $R=0$ occurs near the inner cylinder and then progressively decreases towards the outer cylinder because for this case the temperature of the fluid $T_{f}^{\prime}$ as well as the outer cylinder $T_{b}^{\prime}$ are same and less than the temperature of the inner cylinder $T_{a}^{\prime}$. The maximum velocity occurs in the middle region of the flow for $R=1$ and it shifts towards the inner cylinder as $R$ decreases from 1 to 0 . This phenomenon comes out because for $R>0$, the temperature of fluid $T_{f}^{\prime}$ is less than the temperature of both cylinders $T_{a}^{\prime}$ and $T_{b}^{\prime}$, while for $R=1$ the temperature of both cylinders $T_{a}^{\prime}$ and $T_{b}^{\prime}$ are same but greater than the temperature of fluid $T_{f}^{\prime}$. Also at $R=1$ the flow is symmetric between the cylinders and having the nature as a parabolic type. As the Hartmann number $M$ increases, the velocity profiles have decreasing tendency in the flow region. This suggests that the effect of the buoyancy force parameter can be controlled by increasing the Hartmann number. Fig. 4 is used to demonstrate the velocity profiles for the case $R<0$ when both cylinders are non-conducting. It depicts that the upward flows materialize near the inner cylinder for considered values of $R$ and $M$. At $R=-0.3$, the reverse flow does not appear near the outer cylinder, while it 
occurs for $R=-0.5$ and -0.7 . The magnitude of upward flow increases near the inner cylinder while decreases the magnitude of downward flow near the outer cylinder as $R$ increases. The reason is that for $R<0$, the temperature of fluid $T_{f}^{\prime}$ is greater than the temperature of outer cylinder $T_{b}^{\prime}$ and less than the temperature of inner cylinder $T_{a}^{\prime}$. The influence of Hartmann number is to decrease the magnitude of both upward and downward flows.

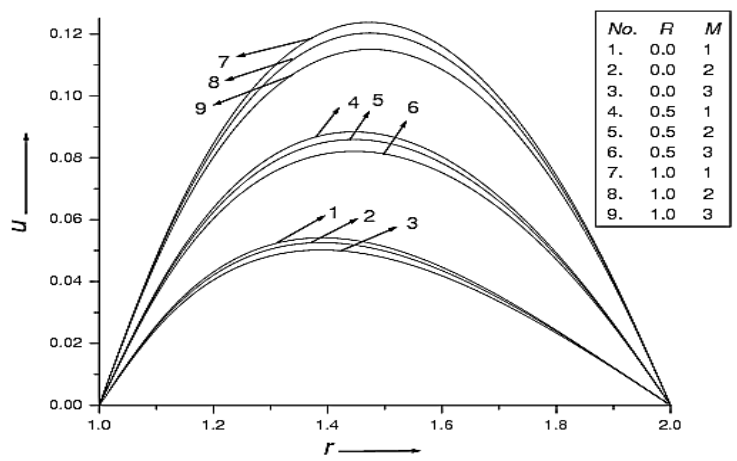

Fig. 3. Velocity profile in case 1 when $0 \leq R \leq 1$ and $\lambda=2.0$

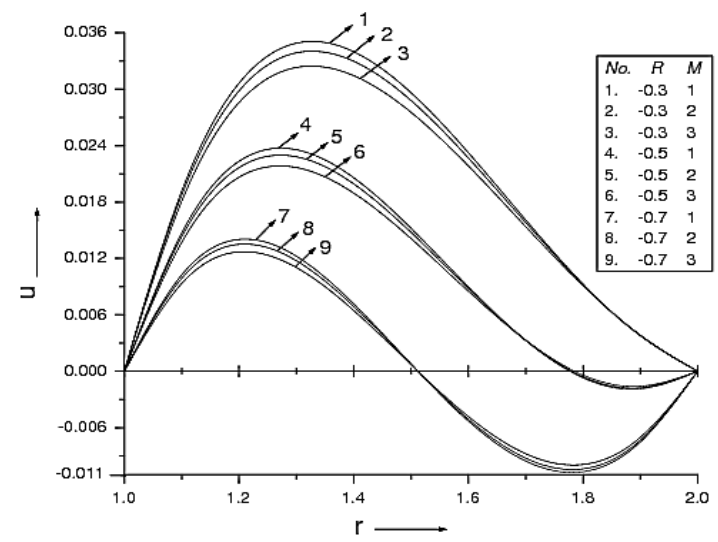

Fig. 4. Velocity profile in case 1 when $R<0$ and $\lambda=2.0$.

Figs. 5 and 6 are used to show the influence of the buoyancy force distribution parameter $R$ on the velocity profiles for the cases $0 \leq R \leq 1$ and $R<0$ respectively when outer cylinder is conducting. The nature of the graphs in these figures is same as Figs. 3 and 4 and the velocity profiles are rapidly decreasing with increase in the value of Hartmann number. A comparative study of Figs. 3 and 5 reveals that the maximum velocity of the fluid in the case when one of the cylinders is conducting is less than the case when both cylinders are non-conducting. The velocity profiles corresponding to the case when the inner and outer cylinders are conducting and non-conducting are not given here for the sake of brevity because obtained results have almost same nature as reported for the case when inner and outer cylinders are non-conducting and conducting.

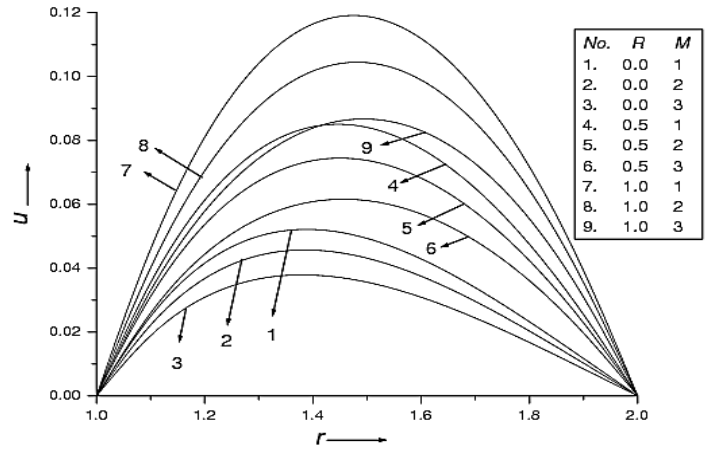

Fig. 5. Velocity profile in case 2 when $0 \leq R \leq 1$ and $\lambda=2.0$.

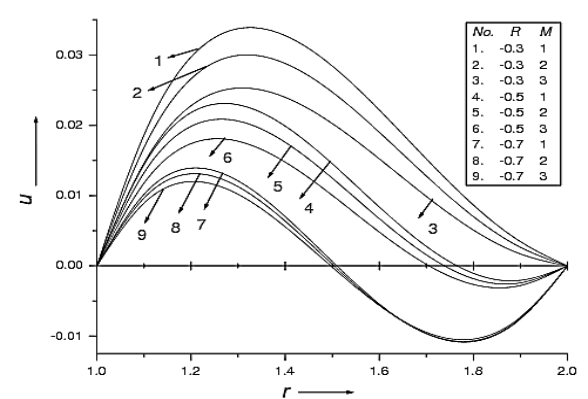

Fig. 6. Velocity profile in case 2 when $R<0$ and $\lambda=2.0$.

Figs. 7 and 8 illustrate the induced magnetic field for the cases $0 \leq R \leq 1$ and $R<0$ respectively when both cylinders are non-conducting. A close study of Fig. 7 suggests that the magnitude of induced magnetic field is maximum in both (upward and downward) directions for $R=1$. The magnitude of induced magnetic field increases near the inner cylinder in upward direction and also near outer cylinder in downward direction as $R$ changes from 0 to 1 . The position of maximum induced magnetic field shifts toward the inner cylinder as $R$ decreases from 1 to 0 . Fig. 8 reveals that the induced magnetic field exits in the upward direction for $R=-0.3$ and -0.5 while at $R=-0.7$ the induced magnetic field does not appear in the upward direction.

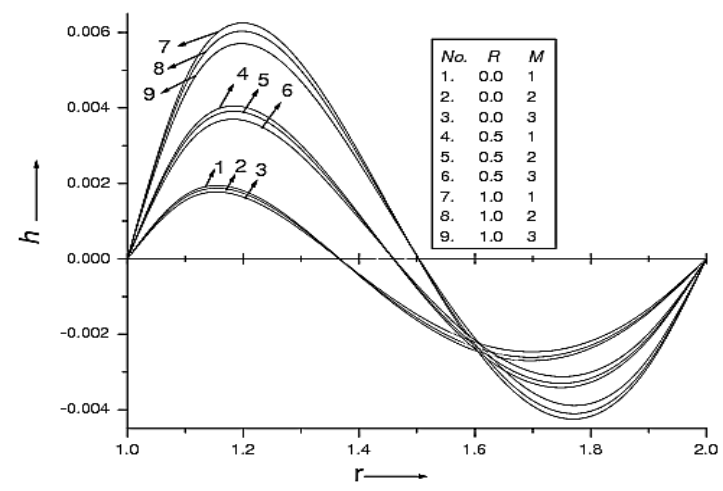

Fig. 7. Induced magnetic profile in case 1 when $0 \leq R \leq$ 1 and $\lambda=2.0$. 


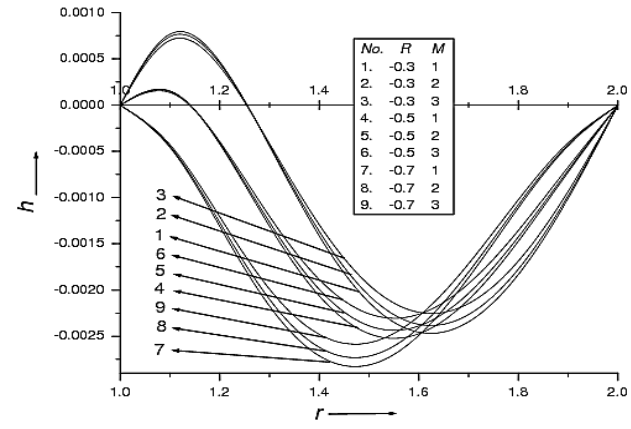

Fig. 8. Induced magnetic profile in case 1 when $R<0$ and $\lambda=2.0$.

In Figs. 9 and 10, we have shown the profiles of induced magnetic for the cases $0 \leq R \leq 1$ and $R<0$ respectively when inner and outer cylinders are non-conducting and conducting. From Fig. 9, we can conclude that the induced magnetic field decreases monotonically in the whole region for all considered values of $R$ and $M$. The magnitude of induced magnetic field increases as buoyancy force distribution parameter $R$ increases. Fig. 10 depicts that the induced magnetic field decreases monotonically for $R=-0.3$ however, at $R=-0.5$, the induced magnetic field decreases monotonically upto $r=1.8$ and thereafter almost constant. Further, the induced magnetic field at $R=-0.7$ decreases monotonically upto $r=1.5$ and then increases monotonically upto the outer cylinder. Thus the point of incredibility of induced magnetic field shifts toward the inner cylinder as $R$ decreases.

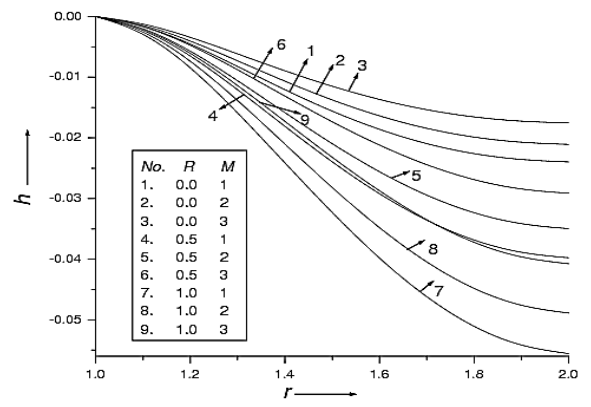

Fig. 9. Induced magnetic profile in case 2 when $0 \leq R \leq$ 1 and $\lambda=2.0$.

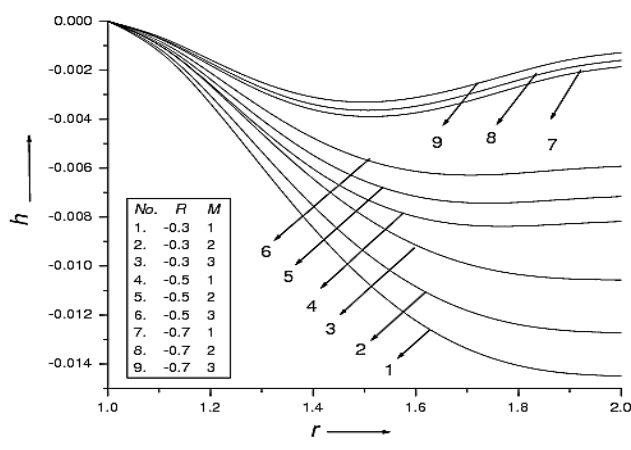

Fig. 10. Induced magnetic profile in case 2 when $R<0$ and $\lambda=2.0$.
Figs. 11 and 12 represent the profiles of induced magnetic field for the cases $0 \leq R \leq 1$ and $R<0$ respectively when inner and outer cylinders are conducting and non-conducting. Fig. 11 clearly shows that the induced magnetic field decreases monotonically from inner cylinder to outer cylinder for all considered values of $R$ and $M$. The maximum induced magnetic field at inner cylinder increase as $R$ changes from 0 to 1 but Hartmann number has reverse effect. A close study of Fig. 12 reveals that the induced magnetic field does not appear in downward direction for $R=-0.3$ while appears for $R=-0.5$ and -0.7 . The magnitude of induced magnetic field decreases monotonically for the values of $R=-0.3$ and -0.5 while for $R=-0.7$ it decreases monotonically upto $r=1.5$ and after it increases monotonically. From a comparative study of the Figs. $7-12$, we find that the magnitude of induced magnetic field is less than the case when both cylinders are non-conducting compared to the case when one of the cylinders is conducting.

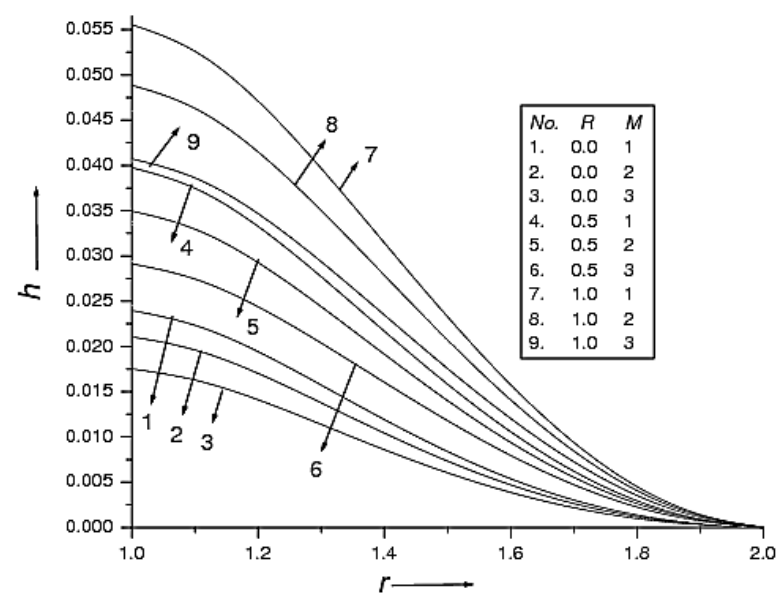

Fig. 11. Induced magnetic profile in case 3 when $0 \leq R \leq$ 1 and $\lambda=2.0$.

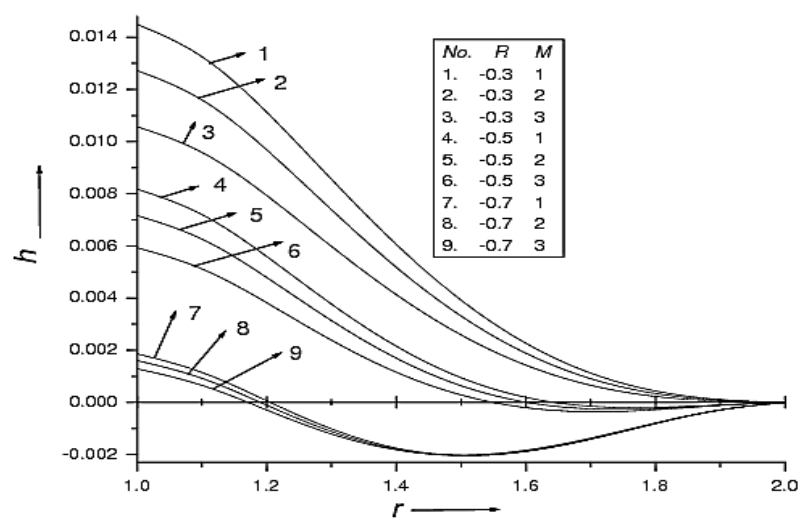

Fig. 12. Induced magnetic profile in case 3 when $R<0$ and $\lambda=2.0$.

Figs. 13 and 14 illustrate the induced current density with respect to $\mathrm{r}$ for the cases $0 \leq R \leq 1$ and $R<0$ 
respectively when both cylinders are non-conducting. Fig. 13 depicts that the positive current density is induced in the middle region of flow while Fig. 14 shows that it is induced near the inner cylinder. The negative current density is induced on the cylinders for both cases. The effect of buoyancy force distribution parameter is to increase the positive current density in the middle region and also the negative current density near the both cylinders. Further, we can observe that the effect of the Hartmann number is to decrease the positive as well as negative induced current density. Through a comparative study of the Figs. 13 and 14 , we conclude that the positive induced current density shifts toward the inner cylinder as $R$ changes from 1 to -0.7. The magnitude of the surface current density is greater at the inner cylinder in comparison to the outer cylinder.

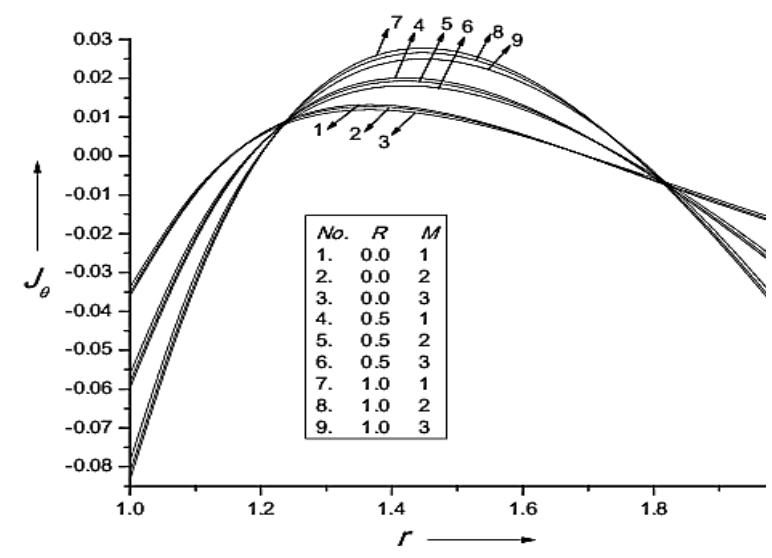

Fig. 13. Induced current density profile in case 1 when $0 \leq R \leq 1$ and $\lambda=2.0$.

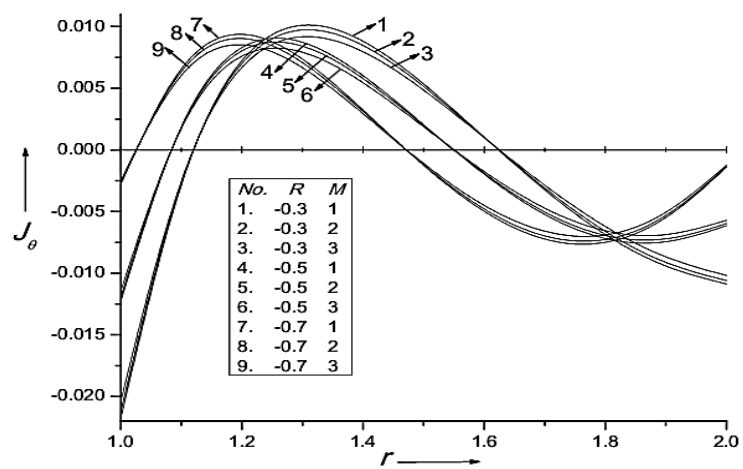

Fig. 14. Induced current density profile in case 1 when $R<0$ and $\lambda=2.0$.

The profiles of induced current density are plotted in Figs. 15 and 16 for the cases $0 \leq R \leq 1$ and $R<0$ respectively when inner and outer cylinders are respectively non-conducting and conducting. These figures depict that the induced current density has rapidly decreasing nature as $M$ increases while increasing nature with $R$. From Fig. 15 it is clear that the maximum current density is induced in the middle region at $R=1.0$ and then it has shifting tendency towards the inner cylinder as
$R$ changes from 1 to 0 . Fig. 16 shows that the positive current density is induced near the inner cylinder. The negative induced current density occurs at outer cylinder for $R=-0.7$ and -0.5 while at $R=-0.3$ it is positive. Comparing the graphs in the Figs. 13 and 15 as well as in Figs. 14 and 16, we find that when outer cylinder is conducting the maximum value of induced current density is greater in comparison to the case when both cylinders are non-conducting. The induced current density corresponding to the case when the inner cylinder is conducting is not reported here for the sake of brevity as having almost the same nature as when outer cylinder is conducting.

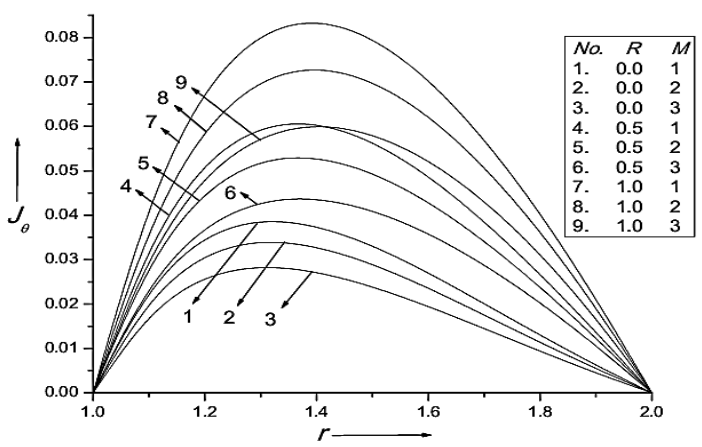

Fig. 15. Induced current density profile in case 2 when $0 \leq R \leq 1$ and $\lambda=2.0$.

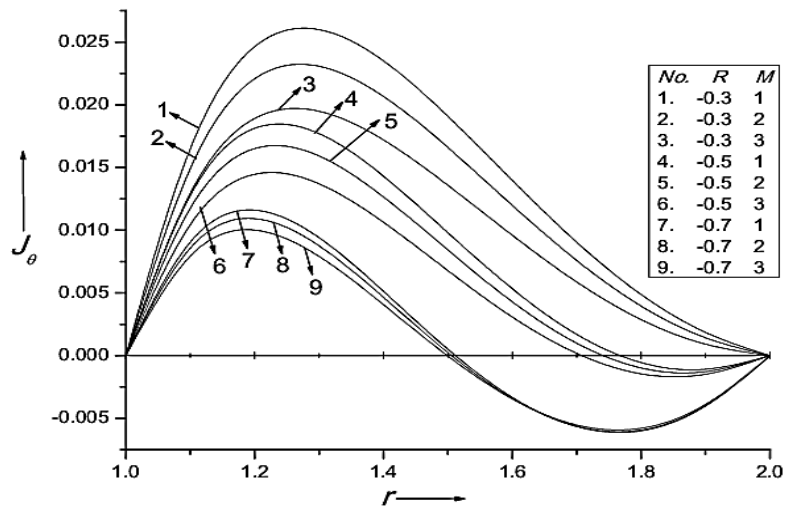

Fig. 16. Induced current density profile in case 2 when $R<0$ and $\lambda=2.0$.

In Fig. 17 we have shown the effect of induced magnetic field on velocity profile at $R=0.0$ by comparing the results corresponding to the cases (i) when induced magnetic field is neglected (Singh et al.(1997)) and (ii) when it is taken into account. It clearly shows that the velocity profiles in the case of considering the induced magnetic field into account is greater in comparison to the case of neglecting the induced magnetic field at the same Hartmann number when both the cylinders are non-conducting. 


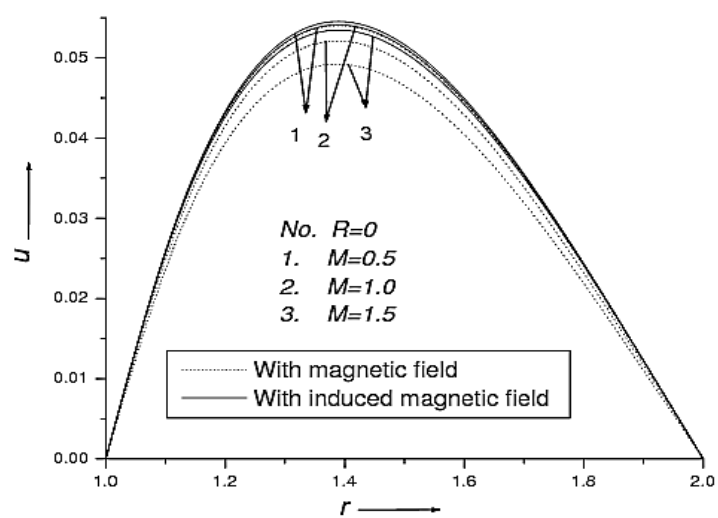

Fig. 17. Effect of induced magnetic field on velocity profile at $R=0.0$ and $\lambda=2.0$

Table 1 represents numerical values of the Nusselt number and it shows that the effect of buoyancy force distribution parameter is to decrease the heat transfer rate on both cylinders. The numerical values of skin-friction $\tau_{1}$ (at the outer surface of inner cylinder), $\tau_{\lambda}$ (at the inner surface of outer cylinder), mass flow rate $Q$ and the induced current flux $J$ are presented in Tables 2 and 3 for the cases 1 and 2 respectively. An observation from these tables is that the numerical value of skin-friction at the outer surface of inner cylinder increases while at the inner surface of outer cylinder decreases as the value of $R$ increases for both cases. The influence of Hartmann number $M$ on the skin-friction at both the cylinders is to decrease for $R \leq 0$, while increase for $0<R \leq 1$ in the case 1 . In case 2 the numerical values of skin-friction at inner cylinder decreases with the values of $M$. As $R$ changes from -0.7 to 1.0 , the difference between the values of $\tau_{1}$ and $\tau_{\lambda}$ increases, which suggests that a desired flow formation can be obtained by assigning a suitable value to $R$. The numerical values of skin-friction at inner cylinder in case 1 is greater than from case 2 while at outer cylinder it is less than from case 2 .

The mass flux $Q$ decreases with increment in $M$ in both cases for considered values of $R$ except at $R=-0.7$. An increase in $R$ leads to reduce the mass flux of fluid $Q$ for both the cases. The increase in values of Hartmann number yields the oscillatory nature in the induced current flux with decreasing amplitude for the case when both cylinders are non-conducting and same type of effect is also due to $R$. In case 2 , the induced current flux increases with $R$ while decreases with $M$. A comparative study of tables 2 and 3 indicates that the mass flux of fluid is grater in the case when both cylinders are non-conducting in comparison to the case when outer cylinder is conducting, while the induced current flux has opposite behavior.

Table 1 Numerical values of non-dimensional Nusselt number at inner and outer cylinders

\begin{tabular}{cccc}
\hline$\lambda$ & $R$ & $N u_{1}$ & $N u_{\lambda}$ \\
\hline 2 & -0.7 & 2.45258157 & 1.22629078 \\
& -0.5 & 2.16404256 & 1.08202128 \\
& -0.3 & 1.87550355 & 0.93775178 \\
& 0.0 & 1.44269504 & 0.72134752 \\
& 0.3 & 1.00988653 & 0.50494326 \\
0.5 & 0.72134752 & 0.36067376 \\
& 0.7 & 0.43280851 & 0.21640426 \\
& & 0.00000000 & 0.00000000 \\
\hline
\end{tabular}

Table 2 Numerical values of non-dimensional skin-friction at inner and outer cylinders in case 1

\begin{tabular}{ccccccc}
\hline$\lambda$ & $M$ & $R$ & $\tau_{1}$ & $\tau_{\lambda}$ & $Q$ & $J$ \\
\hline 2 & 1 & -0.7 & 0.16851794 & 0.11454088 & -0.002705 & $-5.378959 \mathrm{E}-08$ \\
& & -0.5 & 0.21731975 & 0.04714391 & 0.0900505 & $1.011261 \mathrm{E}-07$
\end{tabular}


A. Kumar et al. / JAFM, Vol. 6, No. 1, pp. 15-26, 2013.

\begin{tabular}{|c|c|c|c|c|}
\hline-0.3 & 0.26612156 & -0.02025305 & 0.1828054 & $2.730635 \mathrm{E}-08$ \\
\hline 0.0 & 0.33932428 & -0.12134850 & 0.3219388 & $-8.557370 \mathrm{E}-08$ \\
\hline 0.3 & 0.41252699 & -0.22244395 & 0.4610709 & $-3.578334 \mathrm{E}-08$ \\
\hline 0.5 & 0.46132880 & -0.28984092 & 0.5538264 & 3.794445E-08 \\
\hline 0.7 & 0.51013062 & -0.35723788 & 0.6465819 & $-7.927402 \mathrm{E}-08$ \\
\hline 1.0 & 0.58333333 & -0.45833333 & 0.7857140 & $-2.124928 \mathrm{E}-08$ \\
\hline-0.7 & 0.16415027 & 0.11235704 & -0.0029332 & $-3.937164 \mathrm{E}-09$ \\
\hline-0.5 & 0.21390884 & 0.04543845 & 0.0877608 & $5.994644 \mathrm{E}-09$ \\
\hline-0.3 & 0.26366741 & -0.02148012 & 0.1784548 & $1.660199 \mathrm{E}-08$ \\
\hline 0.0 & 0.33830527 & -0.12185801 & 0.3144958 & $-4.618193 \mathrm{E}-09$ \\
\hline 0.3 & 0.41294312 & -0.22223589 & 0.4505371 & $1.972690 \mathrm{E}-08$ \\
\hline 0.5 & 0.46270169 & -0.28915447 & 0.5412310 & $-1.123315 \mathrm{E}-08$ \\
\hline 0.7 & 0.51246026 & -0.35607306 & 0.6319251 & $-4.132116 \mathrm{E}-09$ \\
\hline 1.0 & 0.58709812 & -0.45645094 & 0.7679660 & $9.523271 \mathrm{E}-10$ \\
\hline-0.7 & 0.15746613 & 0.10901498 & -0.003276 & $-5.887244 \mathrm{E}-09$ \\
\hline-0.5 & 0.20868860 & 0.04282834 & 0.0842638 & $-1.096851 \mathrm{E}-01$ \\
\hline-0.3 & 0.25991107 & -0.02335830 & 0.1718040 & $-2.193332 \mathrm{E}-10$ \\
\hline 0.0 & 0.33674478 & -0.12263825 & 0.3031149 & $-8.509794 \mathrm{E}-09$ \\
\hline 0.3 & 0.41357849 & -0.22191820 & 0.4344252 & 3.448052E-09 \\
\hline 0.5 & 0.46480096 & -0.28810484 & 0.5219656 & $1.419459 \mathrm{E}-08$ \\
\hline 0.7 & 0.51602343 & -0.35429147 & 0.6095058 & $-4.153331 \mathrm{E}-09$ \\
\hline 1.0 & 0.59285714 & -0.45357143 & 0.7408162 & $-1.015472 \mathrm{E}-09$ \\
\hline
\end{tabular}

Table 3 Numerical values of non-dimensional skin-friction at inner and outer cylinders in case 2

\begin{tabular}{ccccccc}
\hline$\lambda$ & $M$ & $R$ & $\tau_{1}$ & $\tau_{\lambda}$ & $Q$ & $J$ \\
\hline 2 & 1 & -0.7 & 0.16758761 & 0.11500604 & -0.003679 & 0.00186059 \\
& & -0.5 & 0.21323090 & 0.04918834 & 0.0857673 & 0.00817765 \\
& & -0.3 & 0.25887419 & -0.01662937 & 0.1752129 & 0.01449479
\end{tabular}


A. Kumar et al. / JAFM, Vol. 6, No. 1, pp. 15-26, 2013.

\begin{tabular}{|c|c|c|c|c|}
\hline 0.0 & 0.32733912 & -0.11535592 & 0.3093833 & 0.02397038 \\
\hline 0.3 & 0.39580405 & -0.21408248 & 0.4435518 & 0.03344591 \\
\hline 0.5 & 0.44144734 & -0.27990018 & 0.5329985 & 0.03976291 \\
\hline 0.7 & 0.48709062 & -0.34571789 & 0.6224450 & 0.04607999 \\
\hline 1.0 & 0.55555556 & -0.44444444 & 0.7566136 & 0.05555556 \\
\hline-0.7 & 0.16095053 & 0.11395691 & -0.006209 & 0.00159982 \\
\hline-0.5 & 0.19958659 & 0.05259958 & 0.0730958 & 0.00716111 \\
\hline-0.3 & 0.23822264 & -0.00875774 & 0.1524006 & 0.01272238 \\
\hline 0.0 & 0.29617672 & -0.10079373 & 0.2713580 & 0.02106422 \\
\hline 0.3 & 0.35413080 & -0.19282973 & 0.3903159 & 0.02940614 \\
\hline 0.5 & 0.39276686 & -0.25418706 & 0.4696208 & 0.03496744 \\
\hline 0.7 & 0.43140291 & -0.31554437 & 0.5489259 & 0.04052874 \\
\hline 1.0 & 0.48935699 & -0.40758030 & 0.6678835 & 0.04887056 \\
\hline-0.7 & 0.15168553 & 0.11190527 & -0.008986 & 0.00128459 \\
\hline-0.5 & 0.18201945 & 0.05616292 & 0.0579211 & 0.00592651 \\
\hline-0.3 & 0.21235336 & 0.00042056 & 0.1248289 & 0.01056836 \\
\hline 0.0 & 0.25785424 & -0.08319298 & 0.2251901 & 0.01753124 \\
\hline 0.3 & 0.30335511 & -0.16680651 & 0.3255515 & 0.02449408 \\
\hline 0.5 & 0.33368902 & -0.22254887 & 0.3924590 & 0.02913599 \\
\hline 0.7 & 0.36402294 & -0.27829123 & 0.4593665 & 0.03377789 \\
\hline 1.0 & 0.40952381 & -0.36190476 & 0.5597278 & 0.04074074 \\
\hline
\end{tabular}

\section{CONCLUSION}

We have theoretically investigated the combined effects of buoyancy force distribution parameter and Hartmann number on the natural convective flow of an electrically conducting fluid in the presence of a radial magnetic field by taking into account the induced magnetic field and imposing mixed types of boundary conditions for the induced magnetic field on the surface of inner and outer cylinders. It is found that the buoyancy force distribution parameter and Hartmann number play an important role in controlling the transport phenomena. The effect of the induced magnetic field is to increase the velocity profiles in comparison to the case of neglecting the induced magnetic field. The effect of Hartmann number is to decrease the magnitude of maximum value of the velocity and induced magnetic field. The decreasing tendency is also observed in the induced current density and mass flux. The influence of buoyancy force distribution parameter is opposite to that of Hartmann number. The magnitude of surface current density is greater at the inner cylinder compared to the outer cylinder. The magnitude of induced magnetic field and current density is greater in the case when one of the cylinders is conducting in comparison to the case when both cylinders are non-conducting. The skin-friction at outer surface of inner cylinder and mass flux of fluid, when both cylinders are non-conducting are greater than the case when one of the cylinders is 
A. Kumar et al. / JAFM, Vol. 6, No. 1, pp. 15-26, 2013.

conducting while the skin-friction at inner surface of outer cylinder and induced current flux have the opposite effect.

\section{REFERENCES}

Chandran, P., Sacheti, N. C. and Singh, A. K., (2001) A unified approach to analytical solution of a hydromagnetic free convection flow, Scientiae Mathematicae Japonicae, 53, 467-476.

Chandran, P., Sacheti, N. C. and Singh, A. K., (1993) Effect of rotation on unsteady hydrodynamic Couette flow, Astrophysics and Space Science, 202, 1-10.

Chandran, P., Sacheti, N. C. and Singh, A. K., (1996) Hydromagnetic flow and heat transfer past a continuously moving porous boundary, International Communications in Heat and Mass transfer, 23, 889-898.

Chandran, P., Sacheti, N. C. and Singh, A. K., (1998) Unsteady hydromagnetic free convection flow with heat flux and accelerated boundary motion, Journal of Physical society of Japan, 67, 124-129.

Dube, S. N., (1971) Magnetohydrodynamic flow between concentric rotating porous cylinders, Def. Sci. J., 21, 253-256.

El-Shaarawi, M. A. I. and Al-Nimb, M. A., (1990) Fully developed laminar natural convection in open ended vertical concentric annuli, International Journal of Heat and Mass Transfer, 33, 1873-1884.

El-Shaarawi, M. A. I. and Sarhan, A., (1981) Developing laminar free-convection in an open ended vertical annulus with rotating inner cylinder, ASME Journal of Heat Transfer, 103, 552-558.

Globe, S., (1959) Laminar steady-state magnetohydrodynamic flow in an annular channel, Physics Fluids, 2 404-407.
Joshi, H. M., (1987) Fully developed natural convection in an isothermal vertical annular duct, International Communication in Heat and Mass Transfer, 14, 657-664.

Lien, F. S., Cha'o-Kung Chen and Chang, Y. M., (1985) Effect of free convection and mass transfer on the flow past an impulsively moving infinite vertical circular cylinder, International Communication in Heat and Mass Transfer, 12, 127-137.

Paul, T. and Singh, A. K. (1998) Natural convection between coaxial vertical cylinders partially filled with a porous material, Forschung im Ingenieurwesen, 64, 157-162.

Pop, I., Ingham, D. B. and Cheng, P., (1993) Transient free-convection about a horizontal circular cylinder in a porous medium, Fluid Dynamics Research, 12, 295-305.

Sastry, V. U. K. and Bhadram, C. V. V., (1978) Hydromagnetic convective heat transfer in vertical pipes, Application Scientific Research, 34, 117-125.

Seong, J. K. and Choung M. L., (2001) Control of flows around a circular cylinder: Suppression of oscillatory lift force, Fluid Dynamics Research, 29, 47-63.

Singh, S. K., Jha, B. K. and Singh A. K., (1997) Natural convection in vertical concentric annuli under a radial magnetic field, Heat and Mass Transfer, 32, 399-401.

Tyvand, P. A., (1995) First order transient free convection about a horizontal cylinder embedded in a porous medium, Fluid Dynamics Research, 15, 277-294.

Yan, B., (2000) Unsteady viscous flow about a submerged circular cylinder with a steady current, Fluid Dynamic Research, 26, 69-94.

\section{APPENDIX A}

$$
\begin{aligned}
& D_{2}=-\frac{2 D_{1}}{\left(8-2 M^{2}\right)}, D_{3}=-\frac{2+D_{1}}{\left(8-2 M^{2}\right)}+\frac{2 D_{1}\left(12-M^{2}\right)}{\left(8-2 M^{2}\right)^{2}}, D_{4}=-\frac{\left(4 D_{3}+4 D_{2}+1\right)}{M^{2}} \\
& D_{5}=-A_{1}-\frac{B_{1}}{M}, D_{6}=-A_{1}-\frac{B_{1}}{M}, D_{7}=-A_{1} D_{11}+B_{1}\left(\frac{8 D_{3}+4 D_{2}+1}{4 M^{2}}\right) \\
& D_{8}=-A_{2} \lambda^{M-1}-\frac{B_{2}}{M} \lambda^{M}, D_{9}=-A_{2} \lambda^{-M-1}+\frac{B_{2}}{M} \lambda^{-M} \\
& D_{10}=\frac{\left(4 D_{2}+D_{1}\right) B_{2} \lambda^{2}}{4 M^{2}}(2 \log \lambda-1)-\frac{\left(2 A_{2}+B_{2} \lambda\right) \lambda}{2}, D_{11}=B_{2} D_{5}-B_{1} D_{8} \\
& D_{12}=B_{2} D_{6}-B_{1} D_{9}, D_{13}=B_{2} D_{7}-B_{1} D_{10}, D_{14}=\lambda^{M}-1, D_{15}=\lambda^{-M}-1 \\
& D_{16}=\left(1-\lambda^{2}\right) D_{3}-D_{2} \lambda^{2} \log \lambda, D_{17}=D_{13} D_{14}-D_{11} D_{16}, D_{18}=D_{12} D_{14}-D_{11} D_{15}
\end{aligned}
$$


A. Kumar et al. / JAFM, Vol. 6, No. 1, pp. 15-26, 2013.

$$
\begin{aligned}
& D_{19}=D_{13} D_{15}-D_{12} D_{16}, C=\frac{D_{17}}{D_{18}}, B=-\frac{D_{19}}{D_{18}}, A=-B-C-D_{3} \\
& D_{20}=B_{1}\left[\frac{4(B-C)-M\left(D_{2}-2 D_{3}\right)}{4 M}\right], D_{21}=\frac{A_{2}}{\lambda}+B_{2} \log \lambda \\
& D_{22}=-A_{2}\left[-\frac{A}{\lambda}-B \lambda^{M-1}-C \lambda^{-M-1}-D_{3} \lambda-D_{2} \lambda \log \lambda\right]-B_{2}\left[A \log \lambda-\frac{B}{M} \lambda^{M}+\frac{C}{M} \lambda^{-M}-\frac{D_{3}}{2} \lambda^{2}+\frac{D_{2}}{4} \lambda^{2}(1-2 \log \lambda)\right] \\
& D_{23}=B_{2} D_{20}-B_{1} D_{22}, D_{24}=A_{1} B_{2}-B_{1} D_{21} \\
& D_{25}=D_{20} D_{21}-A_{1} D_{22}, P=-\frac{D_{25}}{D_{24}}, S=\frac{D_{23}}{D_{24}}
\end{aligned}
$$

\section{APPENDiX B}

$$
\begin{aligned}
& \mathrm{D}_{26}=\frac{-D_{1}}{32}, D_{27}=\frac{-\left(4-D_{1}\right)}{16}, D_{28}=\frac{-D_{1}}{8}, D_{29}=-A_{1}-\frac{B_{1}}{2}, D_{30}=-A_{1}+\frac{B_{1}}{2} \\
& D_{31}=\frac{A_{1}}{8}\left(5 D_{1}-7\right)+\frac{B_{1}}{16}\left(1-D_{1}\right), D_{32}=-A_{2} \lambda-\frac{B_{2}}{2} \lambda^{2}, D_{33}=-A_{2} \lambda^{-3}+\frac{B_{2}}{2} \lambda^{-2} \\
& D_{34}=\frac{A_{2}}{8}\left[\left(5 D_{1}-7\right) \lambda+\left(11 D_{1}-16\right) \lambda \log \lambda-D_{1} \lambda(\log \lambda)^{2}\right] \\
& D_{35}=\frac{B_{2} \lambda^{2}}{16}\left[D_{1}\left\{12 \log \lambda+(\log \lambda)^{2}-1\right\}+(1-16 \log \lambda)\right], D_{36}=D_{34}+D_{35} \\
& D_{37}=B_{2} D_{29}-B_{1} D_{32}, D_{38}=B_{2} D_{30}-B_{1} D_{33}, D_{39}=B_{2} D_{31}-B_{1} D_{36} \\
& D_{40}=\lambda^{2}-1, D_{41}=\lambda^{-2}-1, D_{42}=D_{26} D_{40}+D_{27} \lambda^{2} \log \lambda+D_{28} \lambda^{2}(\log \lambda)^{2} \\
& D_{43}=D_{38} D_{42}-D_{39} D_{41}, D_{44}=D_{38} D_{40}-D_{37} D_{41}, D_{45}=D_{37} D_{42}-D_{39} D_{40} \\
& F=\frac{D_{43}}{D_{44}}, G=\frac{-D_{45}}{D_{44}}, E=-F-G-D_{26}, D_{46}=\frac{D_{26}+D_{28}}{8}, D_{47}=\frac{D_{27}-D_{28}}{2} \\
& D_{48}=\frac{D_{28}}{2}, D_{49}=A_{1}\left[F+G+\frac{\left(D_{26}+D_{27}\right)}{2}\right]+\frac{B_{1}}{2}\left[F-G+\frac{\left(D_{26}+D_{28}\right)}{2}\right] \\
& D_{50}=A_{2}\left[\frac{E}{\lambda}+F \lambda+G \lambda^{-3}+\left(\frac{D_{26}+D_{27}}{2}\right) \lambda+D_{27} \lambda \log \lambda+D_{28} \lambda(\log \lambda)^{2}\right] \\
& D_{51}=B_{2}\left[E \log \lambda+\frac{F}{2} \lambda^{2}-\frac{G}{2} \lambda^{-2}+\left(\frac{D_{26}+D_{28}}{2}\right) \lambda^{2}+\left(\frac{D_{27}-D_{28}}{2}\right) \lambda^{2} \log \lambda+\frac{D_{28}}{2} \lambda^{2}(\log \lambda)^{2}\right] \\
& D_{52}=D_{50}+D_{51}, D_{53}=\frac{A_{2}}{\lambda}+B_{2} \log \lambda, D_{54}=B_{2} D_{49}-B_{1} D_{52} \\
& D_{55}=A_{1} B_{2}-B_{1} D_{55}, D_{56}=D_{49} D_{53}-A_{1} D_{52}, K=-\frac{D_{56}}{D_{55}}, L=\frac{D_{54}}{D_{55}}
\end{aligned}
$$

